\title{
L'obligation de déclarer son patrimoine avant et après l'exercice des fonctions nominatives ou électives en droit positif béninois
}

\author{
Pr. Arsène-Joël Adeloui*
}

«Pour lutter contre la corruption et infractions assimilées dans la fonction publique, les Etats parties s'engagent à exiger que tous les agents publics ou ceux qui sont désignés par les lois nationales déclarent leurs biens lors de leur prise de fonctions, ainsi pendant et à la fin de leur mandat ${ }^{1}$. C'est en ces mots que l'organisation panafricaine a voulu marquer sa détermination pour lutter contre la corruption des agents publics devenue un phénomène complexe et dont les effets sont dévastateurs sur la stabilité politique, économique, sociale et culturelle des pays africains.

Belle initiative à l'échelle continentale, la déclaration de patrimoine est déjà bien comprise par la plupart des Etats africains sortis de la nuit des dictatures qui font de la lutte contre la corruption leur cheval de bataille. En effet, pour garantir un meilleur contrôle des fonds publics, ces Etats ont prévu des dispositions constitutionnelles relatives à la déclaration du patrimoine des agents publics à l'entrée et à la sortie de leurs fonctions. Par cellesci, ils entendent indiquer que les diverses autorités exerçant quelle fonction que ce soit ne doivent pas confondre l'intérêt public avec leurs intérêts privés. L'exigence de transparence $\mathrm{du}$ patrimoine des responsables publics devient dès lors un principe catégorique dont la mise en œuvre suit un régime particulier. Bien que reposant sur un arsenal juridique solide, l'obligation de déclaration du patrimoine se heurte néanmoins à des résistances qui confirment sa portée flexible.

\section{A- La preuve d'un arsenal juridique solide}

La déclaration du patrimoine est une obligation constitutionnelle qui figure en bonne place à l'article 52 alinéa 2 de la Constitution béninoise du 11 décembre 1990 qui dispose: « Le Président de la République et les membres du gouvernement sont tenus, lors de leur entrée en fonction et à la fin de celles-ci, de faire sur l'honneur une déclaration écrite de tous leurs biens et patrimoine adressée à la chambre des comptes de la Cour Suprême ». Cette disposition constitutionnelle porte à la fois sur les titulaires de la déclaration du patrimoine et l'organe compétent du contrôle de cette déclaration.

* Agrégé des Facultés de droit, Maître de conférences des Universités du CAMES, Directeur de l'école doctorale des sciences juridiques, administratives et politiques, Faculté de Droit et de sciences politiques/Université d'Abomey-Calavi

1 Voir art.7.1 de la Convention de l'Union Africaine sur la prévention et la lutte contre la corruption adoptée le 11 juillet 2003 à Maputo (Mozambique). 


\section{I- Les titulaires de la déclaration}

Aux termes de l'article 52.2 précitée, sont concernés par cette mesure le Président de la République, au moment où il entre en fonction et à la fin de celle-ci; mais aussi les membres du Gouvernement à leur nomination et à la cessation de leurs fonctions. En dehors de ces autorités, l'article 3 de la loi n²011- 20 du 12 octobre 2011 portant lutte contre la corruption et autres infractions connexes en République du Bénin élargit la déclaration à d'autres autorités qui exercent des fonctions importantes dans la République et sont appelées à manier les deniers publics. Selon l'article 3 de ladite loi :

"Les hautes personnalités de l'Etat et les hauts fonctionnaires tels que définis par la loi $n^{\circ}$ 2010-05 fixant la liste des hauts fonctionnaires de l'Etat dont la nomination est faite par le Président de la République en conseil des ministres, les directeurs centraux de l'administration, les directeurs des régies financières décentralisées et déconcentrées, les membres des états-majors des armées, les directeurs généraux, les directeurs et cadres de la douane, de la police, de la gendarmerie et des eaux et forêts, les directeurs des offices et sociétés d'Etat, les directeurs/coordonnateurs de projets, les directeurs financiers, les régisseurs, les comptables, les présidents de commissions administratives, les administrateurs d'un ouvrage public ou d'un bien appartenant au domaine de l'Etat, les présidents de tribunaux, juges, procureurs et greffiers et plus généralement tout ordonnateur de dépenses de tout organisme public et de toute personne morale de droit public, les administrateurs, directeurs, comptables et contrôleurs des entreprises publiques et sociétés de droit privé dont le capital est détenu par l'Etat, les ambassadeurs et les membres de l'Autorité nationale de lutte contre la corruption ont l'obligation de déclarer, à la prise et à la fin de service, leur patrimoine.

Ces dispositions s'étendent également aux personnalités élues à un mandat public et à tout agent public de l'Etat dont l'acte de nomination en fait obligation.

Un décret pris en conseil des ministres détermine la liste des hautes personnalités de l'Etat et des hauts fonctionnaires concernés par les présentes dispositions ».

Si l'article 3 précité détermine la liste des personnalités concernées par cette mesure, le décret $n^{\circ} 2012-338$ du 02 octobre 2012 portant modalités d'application des articles 3 et 10 de la loi n²011-20 du 12 octobre 2011 fixe le délai au terme duquel la déclaration de patrimoine doit se faire. Dans son article 7 : «la déclaration de patrimoine est adressée à la juridiction financière compétente dans les quinze (15) jours suivant l'entrée en fonction des hautes personnalités et des hauts fonctionnaires ».

Par devoir de vigilance, les citoyens béninois veillent au strict respect de ces exigences légales. C'est ainsi que deux recours ont été adressés à la Cour constitutionnelle qui s'est prononcée sur le respect de l'article 52 précité.

D'une part, saisie par le requérant Jean-Pierre Y. DEGUE-KAKPO d'un recours contre le président de la République pour violation de l'article 52 qui « a dérogé à son obligation 
constitutionnelle depuis le 06 avril 2016 où il est entré en fonction »; que « le rapport de l'Autorité Nationale de Lutte contre la corruption concluait aussi que douze ministres sur vingt et un(21) ont déclaré leur patrimoine à la date du 31 août 2016 alors qu'au dernier point fait à la date du 17 mai 2016, il n'y avait que quatre(04) ministres qui se sont conformés à cette obligation. Cette obligation, bien que positive, ne doit pas violer le fait qu'aucun ministre ayant déclaré son patrimoine à l'entrée en fonction ne l'a fait dans le délai prévu à l'article 7 du décret $n^{\circ} 2023-338$ du 02 octobre $2012 \ldots$ ce qui constitue une violation flagrante d'une disposition réglementaire par des hautes personnalités de l'Etat. Evidemment, si le chef de l'Etat même ne l'a pas fait, comment ses ministres respecteraient-ils cette disposition constitutionnelle? ».

Pour la Cour constitutionnelle qui s'est fondée sur le point récapitulatif des déclarations de biens et patrimoines des membres du gouvernement et de son chef transmis par la présidente de la chambre des comptes par intérim de la cour suprême, le Président de la République Guillaume Athanase TALON a procédé à la déclaration de ses biens et patrimoine le 06 novembre $2016^{2}$.

Comme on pourrait le constater le délai constitutionnel de déclaration n'a pas été respecté. Il y a eu retard que la Haute juridiction n'a pas condamné puisqu'il semble qu'elle aurait préféré privilégier la déclaration tardive de l'autorité concernée à l'absence de déclaration du patrimoine.

D'autre part, le requérant Faissou Akandé MOUDACHIROU se plaint du comportement de Monsieur Patrice TALON, Président de la République qui a violé son serment de « respecter et de défendre la constitution que le peuple béninois s'est librement donnée ». En effet, son comportement qui consiste à ne pas se conformer à notre constitution et à l'article 7 du décret constitue un mépris à nos lois, alors même que l'article 59 de la constitution dispose : « Le Président de la République assure l'exécution des lois et garantit celle des décisions de justice »; qu'il demande à la Cour « ... de déclarer que ce retard accusé par le président Patrice TALON est une violation de la constitution ». Mais la Cour s'est déclarée incompétente en estimant que les éléments du dossier portent sur un contrôle de légalité du délai de la déclaration du patrimoine conformément aux dispositions des articles 3 et 10 de la loi n ${ }^{\circ} 2011-20$ du 12 octobre 2011 ainsi que de son décret d'application n²02-338 du 02 octobre 2012 portant modalités d'application des articles 3 et 10 de ladite $1 \mathrm{io}^{3}$.

Cette deuxième décision de la Cour apparait ambiguë dans la mesure où le décret fixant le délai n'étant pas a priori contraire à la constitution, le retard accusé par le Président de la République tient lieu d'un mépris des textes qu'on aurait pu sanctionner.

Il y a lieu de relever ici qu'en dehors des autorités gouvernementales, aucune autre autorité de l'Etat n'a pas, à notre connaissance, fait l'objet de recours devant la Cour constitutionnelle pour non-déclaration de ses biens. Bien entendu que c'est l'Autorité Nationale de Lutte contre la corruption (ANLC) qui de façon périodique publie la liste des autorités

2 Cour constitutionnelle du Bénin, DCC 17 -056 du 09 mars 2017.

3 Cour constitutionnelle du Bénin, DCC 17-007 du 25 janvier 2018. 
concernées par cette obligation constitutionnelle qui ne le font pas toujours dans le délai prescrit.

Il est important de rappeler que la situation patrimoniale faisant l'objet de la déclaration comprend les biens et le patrimoine. Il s'agit là de tous les biens appartenant à la personne soumise à déclaration, étendus aux conjoints lorsque cette personne est mariée sous le régime de la communauté des biens.

Par ailleurs, notons que pendant l'exercice de leurs fonctions, ni le Président de la république ni les membres du Gouvernement ne peuvent, pas eux-mêmes ou par intermédiaire, rien acheter ou prendre en bail qui appartienne au domaine de l'Etat, sans autorisation préalable de la Cour constitutionnelle; celle-ci étant compétente pour statuer sur ce cas dans les conditions prévues dans la loi ${ }^{4}$. C'est en application de cette disposition que la Cour constitutionnelle a rendu une décision le 06 janvier 2017 autorisant le Président de la République à acquérir un immeuble de la ville de Cotonou appartenant au domaine privé de l'Etat qui ne serait pas affecté à un service public et sa mise en vente par le ministre en charge du domaine et du foncier ${ }^{5}$. Dans une affaire similaire, la haute juridiction a déclaré nulle et non avenue la cession d'immeuble intervenue le 27 juillet 2009 entre Monsieur Boni YAYI alors président de la République et la Mairie de Parakou. Ladite cession s'étant faite en violation de l'article 52.1 de la constitution, la Cour l'a déclarée contraire à la constitution. Il faut rappeler que c'est sur requête du Maire de Parakou Charles Toko introduit le 05 janvier 2019 que la Cour a rendu la décision DCC 19-1076.

$\mathrm{Au}$ total, la non-déclaration de son patrimoine à l'entrée ou à la sortie des fonctions d'un agent public est une violation de la constitution que l'organe chargé du respect de cette constitution, la cour constitutionnelle sanctionne. Toutefois, cette haute juridiction n'est pas l'organe compétent de contrôle.

\section{II- L 'organe compétent de contrôle}

La déclaration de patrimoine est confiée à la chambre des comptes de la Cour suprême. C'est « la plus haute juridiction de l'Etat en matière administrative, judiciaire et des comptes de l'Etat. Ses décisions ne sont susceptibles d'aucun recours. Elles s'imposent au pouvoir exécutif, au pouvoir législatif, ainsi qu'à toutes les juridictions $»^{7}$. Son Président est nommé pour une durée de cinq ans par le Président de la république, après avis du Président de l'Assemblée nationale, parmi les magistrats et les juristes de haut niveau ayant quinze ans au moins d'expérience professionnelle. La nomination se fait par décret pris en conseil des ministres ${ }^{8}$.

4 Voir Art. 52 1. de la constitution béninoise du 11 décembre 1990.

5 Cour constitutionnelle du BENIN, DCC 17-009 du 06 janvier 2017.

6 Cour constitutionnelle du Bénin, DCC 19-107 du 28 mars 2019.

7 Voir art.131 de la constitution béninoise du 11 décembre 1990.

8 Voir art.133.1 de la constitution béninoise du 11 décembre 1990. 
En matière de contrôle de déclaration de patrimoine, la compétence de la chambre des comptes consiste en l'appréciation des variations qui ont eu lieu et qui lui sont adressées. Elle vérifie qu'il n'y a pas d'enrichissement anormal, incontrôlé.

Aux termes de l'article 4 de la loi $n^{\circ}$ 2011-20 portant lutte contre la corruption et autres infractions connexes en République du Bénin du 30 aout 2011 « La chambre des comptes de la Cour Suprême et les chambres des comptes des cours d'appel sont chargées de recevoir et d'assurer le contrôle des déclarations de patrimoine prévues à l'article 3 .

Ce contrôle doit se faire, tant à l'entrée qu'à la fin des fonctions des personnes visées.

La déclaration de patrimoine est faite par écrit :

- devant la chambre des comptes de la Cour Suprême pour les hautes personnalités de l'Etat et les hauts fonctionnaires;

- devant les chambres des comptes des cours d'appel pour les autres personnes visées à l'article 3 ci-dessus.

Cette déclaration doit être suivie des titres prouvant la propriété des déclarants.

En cas de dissimulation ou de fausses déclarations, l'agent concerné est puni conformément aux dispositions de la présente loi.

Le refus de déclaration est puni d'une amende dont le montant est égal à six (06) mois de rémunération perçue ou à recevoir dans la fonction occupée.

L'amende est prononcée d'office ou sur dénonciation par le président de la Chambre des comptes suivant la distinction établie à l'alinéa 3 ci-dessus »".

Cette disposition indique la procédure à suivre par chaque personne concernée pour déclarer son patrimoine et les sanctions infligées en cas de non-respect de la précédente.

Il est à regretter que jusque-là les fausses déclarations ou la non déclaration par certaines autorités dans les conditions précédemment rappelées ne donnent pas encore lieu à des sanctions appropriées. Ce qui laisse penser que cette obligation est un vœu pieux qui porte un grave préjudice au respect du bien public

\section{B- $L$ 'épreuve de déclaration du patrimoine}

L'obligation de déclaration est éprouvée parce qu'elle contient des failles qui peuvent être néanmoins surmontées.

\section{I- Les failles révélées}

Elles sont essentiellement textuelles en raison de l'ambiguïté du cadre juridique. Les obligations qui pèsent ainsi sur les titulaires des fonctions politiques ne permettent pas de vérifier s'il y a enrichissement ou non. En effet, cette formalité n'a de sens que si les déclarations présentées à la chambre des comptes de la Cour suprême sont sincères et authentiques. S'il n'y a pas de pouvoir d'investigation, ne sera contrôle et vérifié que ce qui a été déclaré.

Ensuite, il est important de noter que le patrimoine ne peut pas être considéré comme le seul critère d'enrichissement possible. D'autres moyens existent pour s'enrichir tels que 
l'attribution clandestine d'avantages en nature permettant un accroissement anormal du grain de vie et qui échappe à la déclaration.

Ainsi, l'objectif de transparence n'est pas garanti s'il porte uniquement sur une déclaration axée exclusivement sur le patrimoine.

Considérons que la déclaration couvre le patrimoine de la personne et de son conjoint que s'ils sont sous le régime de la communauté des biens. S'ils sont sous le régime de la séparation, la déclaration ne comportera pas le patrimoine du conjoint. Enfin, cette déclaration devrait s'étendre à tous les autres membres de la famille, par exemple, les enfants.

Les difficultés que rencontre la mise en ouvre de cette obligation peuvent être transformées en des défis à relever

\section{II- Les solutions envisagées}

Un certain nombre de mesures permettraient de répondre au souci de transparence. L'obligation de déclaration pourrait être étendue à toute personne détenant une parcelle de pouvoir quelle qu'il soit dans le secteur public ou parapublic. Le principe de déclaration pourrait être instauré de façon annuelle. La constitution prévoit l'obligation de déclaration en début et fin de fonction. Il faut prendre en compte le fait que le patrimoine peut faire l'objet d'une variation d'une année à une autre.

La déclaration ne porte que sur le patrimoine, elle pourrait être étendue à la connaissance des revenus des hommes politiques, ce qui permettrait de mieux comprendre l'origine des variations de patrimoine. Une définition rigoureuse des éléments constitutifs du patrimoine et des modalités de leur évaluation devrait être instaurée. Enfin, il est nécessaire que les délais de déclaration soient appliqués rigoureusement. Ils peuvent même être prolongés pour que la déclaration soit la plus complète possible 9 . Enfin, il serait bien d'envisager de sanctions équivalentes à toute autorité qui fait de déclaration tardive, de fausse déclaration, de déclaration partielle à l'entrée ou à la sortie des fonctions ou qui n'en fait pas du tout.

Le rôle joué par l'Autorité Nationale de Lutte contre la Corruption devient très important car de par ses rapports on est désormais situé sur l'évolution périodique de la déclaration du patrimoine faite par les autorités. Dans son dernier rapport publié ne mai 2019 prenant en compte la situation de déclaration de patrimoine au niveau des institutions à la date du 04 avril 2019, l'ANLC fait le point suivant :

- vingt-trois (23) déclarations de patrimoine à l'entrée en fonction ont été enregistrées sur la période allant du 1 er janvier au 04 avril 2019 provenant des cadres des ministères.

- Au 04 avril 2019, tous les vingt-deux (22) membres du Gouvernement mis en place par le décret n²018-198 du 05 juin 2018 ont satisfait à l'obligation de déclaration de patrimoine.

- A l'Assemblée nationale, aucune déclaration de patrimoine n'a été enregistrée sur la période ' $1{ }^{\mathrm{er}}$ janvier au 04 avril 2019. Au total, Soixante-quatorze (74) des quatre-vingt-

9 P.A., LORENZI, Corruption et imposture, Paris, Balland, 1995, 262p. 
deux (82) députés de l'Assemblée Nationale, 7ème législature, ont satisfait à l'obligation légale de déclaration de patrimoine à la date du 04 avril 2019.

- A la Cour constitutionnelle, tous les sept (07) sages de la sixième mandature de la Cour Constitutionnelle ont déclaré leur patrimoine à l'entrée en fonction.

- A la Cour suprême, aucune déclaration de patrimoine n'a été enregistrée sur la période considérée au niveau de cette institution ( $1^{\text {er }}$ janvier au 04 avril 2019).

- A la Haute Cour de Justice, tous les juges de cette institution ont déclaré leur patrimoine à leur entrée en fonction.

- Au Conseil Economique et Social, vingt-sept (27) conseillers sur trente (30) ont déclaré leur patrimoine à l'entrée en fonction.

- A la Haute Autorité de l'Audio-visuel et de la communication, tous les neuf (09) membres de la Haute Autorité de l'Audiovisuel et de la Communication ont déclaré leur patrimoine.

- A la Commission Electorale Nationale Autonome, tous les membres de la Commission Electorale Nationale Autonome ainsi que le Secrétaire permanent de l'organe ont satisfait à l'obligation de déclaration de patrimoine à leur entrée en fonction.

- A l'Autorité Nationale de Lutte contre la corruption, tous les membres de la deuxième mandature de l'institution ont déclaré leur patrimoine à leur entrée en fonction.

- A l'Autorité de régulation des marchés publics, tous les onze (11) membres de l'actuelle mandature de l'ARMP ont procédé à la déclaration de leur patrimoine à leur entrée en fonction.

- A l'Autorité de régulation des communications électroniques et de la Poste (ARCEP), tous les neuf (09) conseillers nommés de l'actuelle mandature de l'ARCEP ont déclaré leur patrimoine à l'entrée en fonction.

Globalement, des efforts sont déployés de part et d'autre pour respecter l'obligation constitutionnelle de déclarer son patrimoine. Le pari n'est pas encore gagné. Le retard comme le défaut de déclaration, les fausses déclarations et le manque de sanctions équivalentes constituent aujourd'hui les angles morts de l'effectivité de la procédure. C'est pour cette raison que les organes chargés de veiller au respect des textes doivent rester en permanence vigilants. Car, comme on le sait, la lutte contre la corruption est un bon indicateur de la bonne gouvernance, et l'obligation de déclaration de patrimoine est une mesure qui participe au renforcement de l'Etat de droit. 


\section{BIBLIOGRAPHIE}

Constitution de la république du Bénin du 11 décembre 1990

Cour constitutionnelle du Bénin, DCC 19-107 du 28 mars 2019

Cour constitutionnelle du Bénin, DCC 17-007 du 25 janvier 2018

Cour constitutionnelle du Bénin, DCC 17 -056 du 09 mars 2017

Cour constitutionnelle du BENIN, DCC 17-009 du 06 janvier 2017

Convention de l'Union Africaine sur la prévention et la lutte contre la corruption adoptée le 11 juillet 2003 à Maputo (Mozambique)

LORENZI, P.A., Corruption et imposture, Paris, Balland, 1995 